\title{
Friction and Wear Mechanisms of Cu/ta-C Coatings Under PAO-4 and PAO-4 with MoDTC Lubrication
}

\author{
Decelyne Elly Binjua ${ }^{1}$, Seock-Sam Kim ${ }^{1 *}$, Young-Jun Jang ${ }^{2}$ and Jong-Kuk Kim ${ }^{2}$ \\ ${ }^{1}$ Department of Mechanical Engineering, Universiti Malaysia Sabah, Malaysia \\ ${ }^{2}$ Department of Extreme Environmental Coatings, Korea Institute of Materials Science, South Korea
}

Received: November 26, 2020, Revised: December 17, 2020, Accepted: December 17, 2020, Available Online: December 23,2020

\begin{abstract}
The tribological behavior of various types of DLC coatings in formulated and non-formulated lubricants are needed for proper usage of these coatings. In this research, the friction and wear mechanism of four different DLC coatings in poly-alpha-olefin type 4 (PAO-4) with and without MoDTC were investigated using ball-on-disc tribometer. One ta-C (tetrahedral amorphous carbon) and three $\mathrm{Cu} /$ ta-C (copper doped ta-C) with different sputter power of $50 \mathrm{~W}, 150 \mathrm{~W}$, and $200 \mathrm{~W}$ coatings were deposited on silicon wafers by using FCVA (filtered cathodic vacuum arc) technique for this research. The results indicate that ta-C coating on silicon wafer has the lowest average friction coefficient $(\mathrm{CoF})$ and better wear resistance than $\mathrm{Cu} / \mathrm{ta}-\mathrm{C}$ coating when lubricated under PAO-4 oil with MoDTC. Cu/ta-C with sputter powers of $150 \mathrm{~W}$ and $200 \mathrm{~W}$ exhibited the highest average friction coefficient under PAO-4 oil with MoDTC. Meanwhile, the average $\mathrm{CoF}$ for all samples were similar under PAO-4 base oil. In terms of wear, ta-C coating showed the highest wear rate under PAO-4 base oil then followed by $\mathrm{Cu} / \mathrm{ta}-\mathrm{C}$ with sputter power of $50 \mathrm{~W}$. Nonetheless, $\mathrm{Cu} / \mathrm{ta}-\mathrm{C}$ with sputter powers of $150 \mathrm{~W}$ and $200 \mathrm{~W}$ exhibited significantly low wear rate under PAO-4 base oil compared to PAO-4 oil with MoDTC.
\end{abstract}

Keywords: Friction; Wear; Cu/ta-C coating; PAO-4; MoDTC; FCVA.

This work is licensed under a Creative Commons Attribution-Non Commercial 4.0 International License.

\section{Introduction}

In order to deliver greater wear resistance, coating materials with high hardness were developed to fulfil the demand for increased durability of mechanical components used in harsh environments. This type of coating has been demanded extensively for various engineering applications.

Diamond-like Carbon (DLC) coating is a coating that possesses properties of natural diamond with mechanical properties such as high wear resistance, high hardness, and a low coefficient of friction under dry conditions. DLC coatings are constructed by a network of different fractions of $\mathrm{sp} 2$ and $\mathrm{sp} 3$ hybridized $\mathrm{C}-\mathrm{C}$ and $\mathrm{C}-\mathrm{H}$ bonding and are usually amorphous [1].

Plasma Enhanced Chemical Vapour Deposition (PE-CVD) is an amorphous film used to coat a protective layer from gas state to solid state on a substrate. The deposition pressures influence the structures and properties of coatings. The average thickness of the coating increased as the deposition pressure increased. According to X. Wang et al. [2], in order to promptly deposit DLC coating, a pipeline is used directly as a deposition chamber by exciting carbon-containing source gas. DLC coating has excellent properties which is a good prospect in the field of inner protective coating. Application of coatings is one of the most widely used route in order to tailor wear performance, surface morphology, fatigue strength and adhesion of substrate material without altering bulk properties of the substrate.

Many coated DLC parts are used in bigger tribological system where steel-steel contacts still exist hence the coating performance under lubricated conditions becomes an important factor. DLC coating can be used for a variety of applications due to the modifications that can be done and its properties such as chemical inertness, infrared transparency, high electrical resistivity, high wear resistance and low friction coefficients that can be adjusted by choosing the right deposition method.

There are many deposition methods of DLC films. Filtered cathodic vacuum arc (FCVA) is one of the deposition processes used to deposit thin films for a variety of applications. This technique can filter the macroparticle during the deposition process by using the magnetic filters in order to produce tremendously high quality of ta-C coatings with $87 \%$ of sp3 bonding fraction. Hence, producing coatings with outstanding properties and are suitable to enhance the performance in corrosion and wear resistance [3].

However, the number of industrial utilisations of DLC films is limited due to their low toughness that causes the films to become brittle. Many have used metal doping to modify the microstructure of the DLC films to solve this problem. Copper is known to be a ductile metal and non-carbide former which can be used as the doping element to enhance the friction and wear mechanisms of DLC coatings under lubrication [4]. In this research, $\mathrm{Cu}$ was doped into ta-C films with different concentration by changing the sputter power.

Moreover, Molybdenum Dithiocarbamate or well known as MoDTC is a complex additive package that can be mixed into the base oil for better performance of lubricants.

Poly-alpha-olefin (PAO-4) base oil is one of the synthetic hydrocarbon liquids with low comparatively short molecular 
lengths and side branch densities that were established as highperformance base-stock oils for automotive and industrial applications. Thereby, this research paper aims to investigate ta$\mathrm{C}$ and $\mathrm{Cu} / \mathrm{ta}-\mathrm{C}$ with different sputter power coatings on silicon wafer under PAO-4 oil with MoDTC and PAO-4 base oil lubrications.

\section{System Design}

In order to successfully execute the experiment, a set of systematic method is required to be set as a standard. The methods for data collection are included as well as the concepts and theories which underline the methods. The experiments procedures, concepts and theories are described in detail for further understanding on the experiment.

\subsection{Deposition Process}

In order to systematically study ta-C films under lubrication, one set of ta-C and three sets of $\mathrm{Cu}$ doped ta-C with different sputter power coated silicon wafer samples were designed. Three main deposition processes which are etching, sputtering and arc deposition were performed to fabricate the coated samples.

The silicon wafers undergo etching process by using the linear ion source (LIS) for 10 minutes to remove the impurities on the surface of the silicon wafers beforehand. Argon gas is used as the reactant gas with a flow of $18 \mathrm{sccm}$. The voltage supply used was $1.7 \mathrm{kV}$ which yielded a $260 \mathrm{~mA}$ of current.

After the etching process, it was then continued with the sputtering of $\mathrm{Cu}$ for 30 minutes to deposit a buffer layer on the surface of the substrate to enhance the adhesion strength of the coating layer. Argon gas was used as the reactant gas with a flow of $25 \mathrm{sccm}$ and a power supply of $250 \mathrm{~W}$.

Then, as for the coating layer, two types of coatings were designed which are ta- $\mathrm{C}$ and $\mathrm{Cu}$ doped ta- $\mathrm{C}$ coatings in this research. There were two processes involved in this coating process which are arc deposition and sputter processes. Both coatings have the same thickness which are $1 \mu \mathrm{m}$ but different deposition time. Arc deposition process was used to deposit the ta-C film on the substrates, meanwhile, arc deposition and sputter processes were used simultaneously to produce $\mathrm{Cu}$ doped ta- $\mathrm{C}$ film. The arc deposition process was conducted under duct bias voltage of $10 \mathrm{~V}$ with a reference current of $60 \mathrm{~A}$ in a $45^{\circ}$-bend magnetic FCVA system for both types of coatings.

For the $\mathrm{Cu}$ doped ta- $\mathrm{C}$ coating, it was conducted with argon gas as the source of reactant gas with a flow of $25 \mathrm{sccm}$. The power supply used varies for the $\mathrm{Cu}$ doped ta-C coating as there were three different samples designed. There are four samples that were designed with their own specific condition where one sample is for ta-C coating and the other three samples are for $\mathrm{Cu} /$ ta-C coating with variation of sputter power as shown in Table 1 below which reveals the coating layer structure of the substrate.

Table 1 The Type of ta-C and $\mathrm{Cu}$ Doped ta-C Coatings

\begin{tabular}{|l|l|l|l|l|}
\hline Thickness & Sample \#1 & Sample \#2 & Sample \#3 & Sample \#4 \\
\hline $1 \mu \mathrm{m}$ & ta-C & $\begin{array}{l}\mathrm{Cu} / \mathrm{ta}-\mathrm{C} \\
(50 \mathrm{~W})\end{array}$ & $\begin{array}{l}\mathrm{Cu} / \mathrm{ta}-\mathrm{C} \\
(150 \mathrm{~W})\end{array}$ & $\begin{array}{l}\mathrm{Cu} / \mathrm{ta}-\mathrm{C} \\
(200 \mathrm{~W})\end{array}$ \\
\hline $0.3 \mu \mathrm{m}$ & \multicolumn{3}{|c|}{ Cu Buffer Layer } \\
\hline \multicolumn{4}{|c|}{ Silicon wafer (100) } \\
\hline
\end{tabular}

\subsection{Tribological Test}

The sliding friction test was carried out by using a ball-ondisk tribometer to determine the friction and wear properties of the coating film.

The counter specimen used was silicon nitride (Si3N4) ball of $4 \mathrm{~mm}$ radius. Prior to the experiments, the ball was cleaned in an ultrasonic bath using ethanol for 3 minutes to remove surface contaminants on the ball. The zig used to hold the silicon wafer was also cleaned by using ethanol. Then, the silicon wafer was attached to the zig with a glue.

Four types of samples with different deposition parameters were used for this experiment. The ball is reciprocated for 1 hour and 39 minutes against the sample with an applied load of $20 \mathrm{~N}$, a linear speed of $84 \mathrm{~mm} / \mathrm{s}$ and a stroke length of $4 \mathrm{~mm}$ at ambient temperature of $18-23{ }^{\circ} \mathrm{C}$ with humidity of $10-20 \%$ for a total distance of $502654.82 \mathrm{~mm}$ of 20000 cycles under two types of lubrications which are PAO-4 base oil and PAO-4 oil with MoDTC.

The coefficient of friction $(\mathrm{CoF})$ was monitored as a function of the number of cycles. The results of friction coefficient were obtained by referring to the real time display of test information in computer software of tribometer. The average friction coefficient of each tested sample was computed and plotted in a graph for both lubrications. The wear rate for each tested sample was also recorded and plotted in a graph. Then, the images of the wear scar of the counterpart material and the wear track on the coating surface were observed by using confocal microscopy.

\section{Results and Discussions}

The results and discussions are presented in four subsections according to coating characterization, friction behavior, wear behavior and surface analysis.

\subsection{Coating Characterization}

The coating characterization was distinguished by comparing results from the previous study. Fig. 1 shows the $\mathrm{Cu}$ concentration and the average growth rate of the films as a function of the sputtering current of the magnetron sputtering unit. The $\mathrm{Cu}$ concentration increased with the sputtering current. In this research, the sputtering power was adjusted to control the $\mathrm{Cu}$ concentration in films. The sputtering current increased with the sputtering power, indicating that the $\mathrm{Cu}$ concentration increases when higher sputtering power is used.

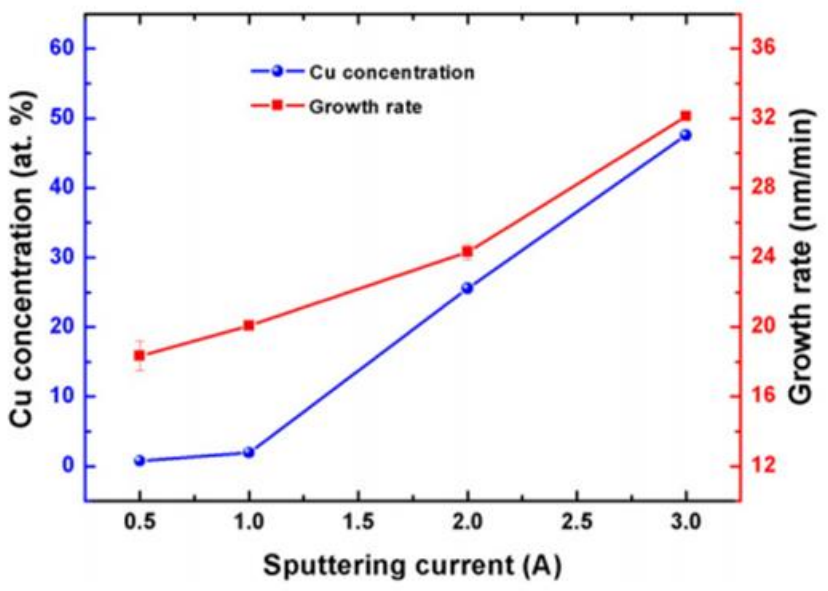

Fig. $1 \mathrm{Cu}$ concentration and growth rate of the $\mathrm{Cu}-\mathrm{DLC}$ films as a function of the sputtering current. (Source: [4]) 
Moreover, the residual stresses of the films were measured as a function of the $\mathrm{Cu}$ concentration, as shown in Fig. 2. Based on previous study, pure DLC film has higher residual stress which is about $2.7 \mathrm{GPa}$ while the residual stress for $\mathrm{Cu}$-DLC film is lower with $1.4 \mathrm{GPa}$ when $\mathrm{Cu} 0.74$ at.\%. The residual stress of the films increases with increasing $\mathrm{Cu}$ concentration and starts to reduce when $\mathrm{Cu}$ concentration is 25.6 at.\% which can be seen from Fig. 3. Thereby, it is believed that the residual stresses in this research study are similar to the previous study whereas it increases as the sputtering power increases.

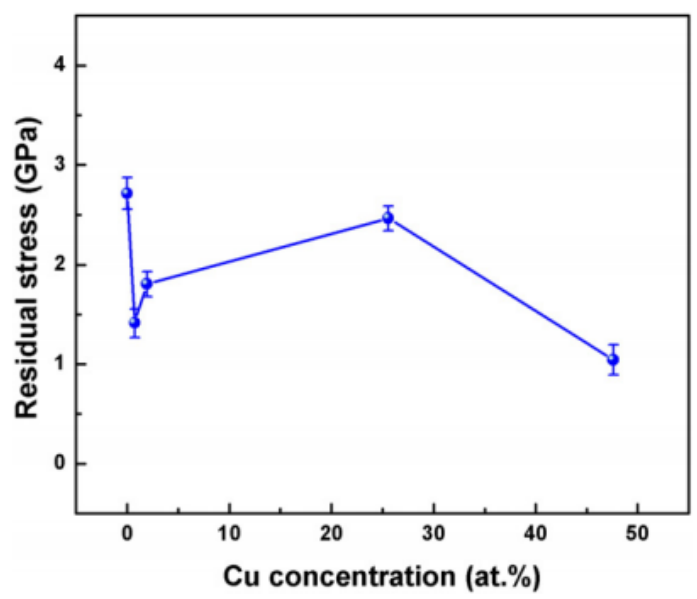

Fig. 2 Residual stress of the Cu-DLC films as a function of $\mathrm{Cu}$ concentration. (Source: [4])

The graph pattern of the film hardness and elastic modulus at different $\mathrm{Cu}$ concentrations were similar to the residual stress as both the mechanical properties and residual stress of the DLC films were highly dependent on the sp3-C bonding structures [4]. The film shows lower hardness compared to pure DLC at lower concentration of $\mathrm{Cu}$. This result is homogenous to the previous research conducted by Hojun Ryu et al. [5], which showed that those $\mathrm{N}$-doped ta-C films have lower hardness than that of nondoped ta-C film. Thus, it can be concluded that non-doped ta-C has higher hardness compared to $\mathrm{Cu}$-doped ta-C in this study.

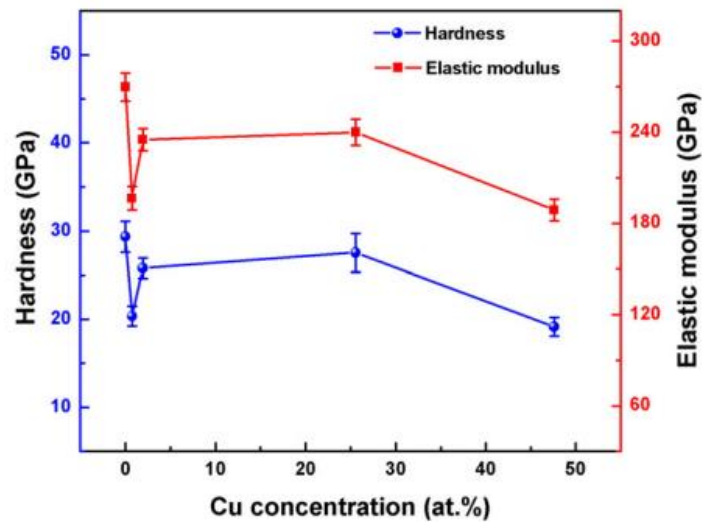

Fig. 3 Hardness and elastic modulus of the $\mathrm{Cu}-\mathrm{DLC}$ films as a function of $\mathrm{Cu}$ concentration. (Source: Dai et al. 2015)

\subsection{Frictional Behaviors Under PAO-4 Base Oil and PAO-4 Oil with MoDTC Lubrications}

This sliding friction test was carried out by using ball-ondisc tribometer to determine the friction and wear properties of the coating film under two different types of lubrication which are PAO-4 base Oil and PAO-4 Oil with MoDTC.
Normally, PAO is known to have better lubricity hence the reduced friction coefficient. Moreover, the friction reduction might be due to the formation of MoDTC derived tribofilms MoS2 and surface graphitization [6], [7]. The addition of MoDTC further improve the friction performance. According to Zahid et al. [8] the surface energy is improved when DLC coatings are doped with metals as the chemical reactivity of the coating with lubricant is increased due to formation of active sites.

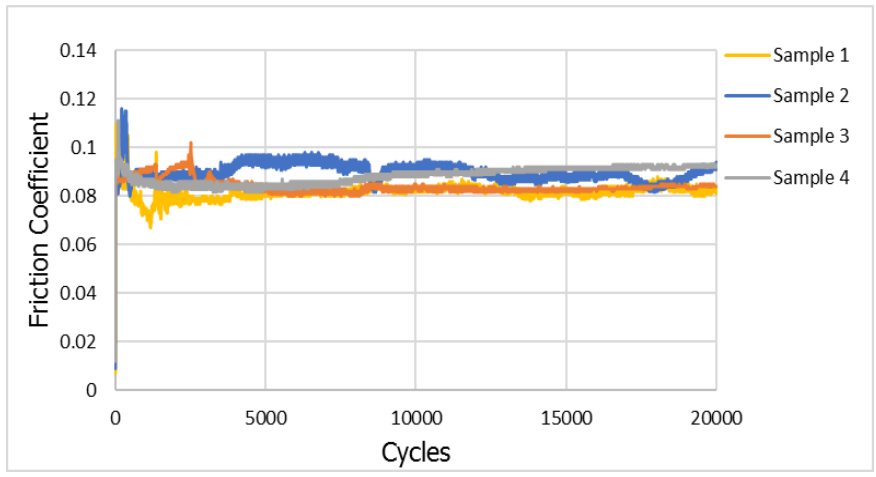

Fig. 4 Frictional behavior of ta- $\mathrm{C}$ and $\mathrm{Cu}$-doped ta- $\mathrm{C}$ coatings under PAO-4 Base Oil

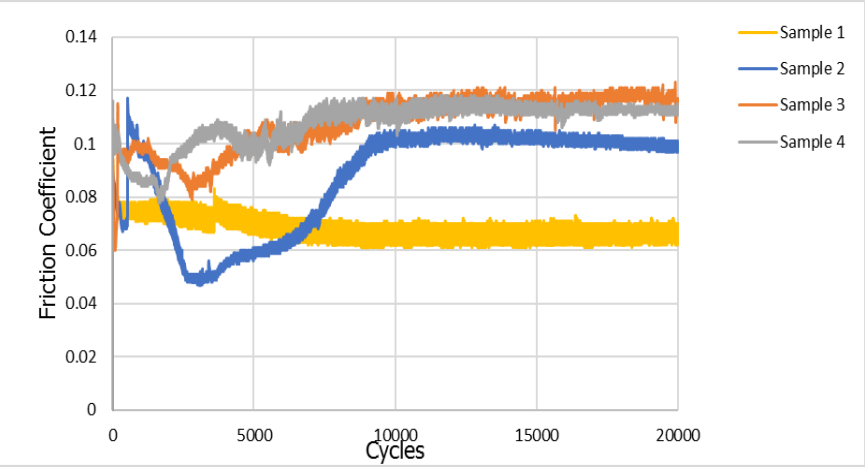

Fig. 5 Frictional behavior of different type of ta-C and $\mathrm{Cu}-$ doped ta-C coating under PAO-4 with MoDTC

Fig. 4 shows the behavior of the $\mathrm{CoF}$ for ta- $\mathrm{C}$ and $\mathrm{Cu}$ doped ta-C coating in PAO-4 base oil lubrication. In the case of ta-C coating, the coefficient of friction was the lowest and showed stable behavior, but it was confirmed that the coefficient of friction was lowered as the UBM (Unbalanced Magnetron) power for $\mathrm{Cu}$ doping increased. This is due to the high hardness characteristics of the ta-C coating film, and it is believed that the contact area is relatively smaller than that of $\mathrm{Cu}$ doping ta- $\mathrm{C}$. The mechanical properties of the ta-C coating used in the experiment can be confirmed from the previous results [9]. In addition, the $\mathrm{Cu}$-doped ta-C coating exhibits a high coefficient of friction behavior when contacting the mating material with the two materials ta- $\mathrm{C}$ and $\mathrm{Cu}$ during friction.

In addition, as shown in Fig. 5, when PAO-4 base oil with MoDTC was used, the ta-C coating still showed the lowest coefficient of friction, but the $\mathrm{Cu}$ doping ta- $\mathrm{C}$ showed a high friction behavior of $0.08 \sim 0.1$ or more. However, it can be seen that the friction coefficient characteristics increase as the $\mathrm{Cu}$ sputter power increases.

The $\mathrm{Cu}$ sputter power of $50 \mathrm{~W}$ showed the lowest coefficient of friction among $\mathrm{Cu}$-doped ta-C, which is believed to be due to the doping of a small amount of $\mathrm{Cu}$ compared to $150 \mathrm{~W}$ and 200 W. 
3.3 Wear Behaviors Under PAO-4 Base Oil and PAO-4 Oil with MoDTC Lubrications

The wear behavior of disk and counter materials was investigated based on the analysis of frictional behavior of ta-C coating and $\mathrm{Cu}$ doped ta-C coatings. Fig. 6 and Fig. 7 show the amount of wear of the disk and the counter materials (Si3N4) used in the PAO-4 base oil and PAO-4 oil with MoDTC lubrications.

In the case of ta-C coating, the wear of disk and Si3N4 ball was highest in PAO environment. This is believed to be due to the simultaneous wear of the counterpart material and disk during friction due to the high hardness characteristics of the ta$\mathrm{C}$ coating without the chemical effect of oil. In addition, it can be understood that carbon-based particles wear due to abrasion have a lubrication effect at the contact interface, so that the amount of wear is high, but the coefficient of friction is low.

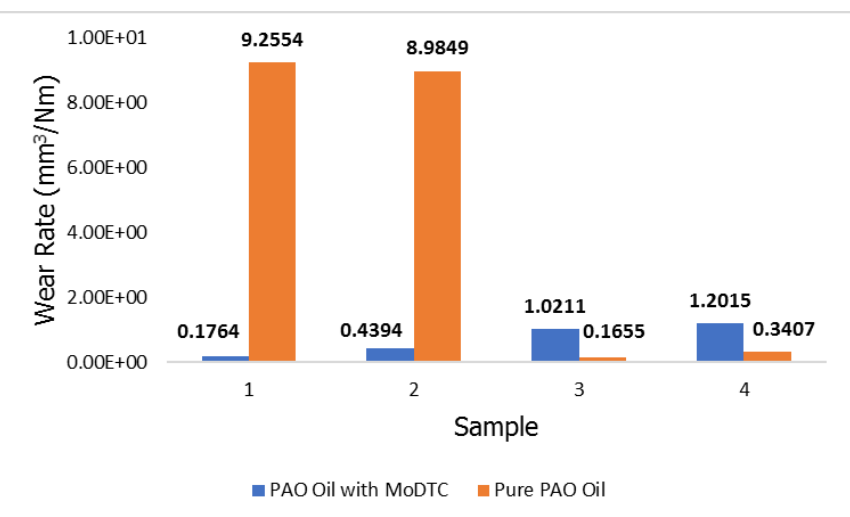

Fig. 6 To compare with wear rate of coatings under the PAO-4 base Oil and PAO-4 with MoDTC

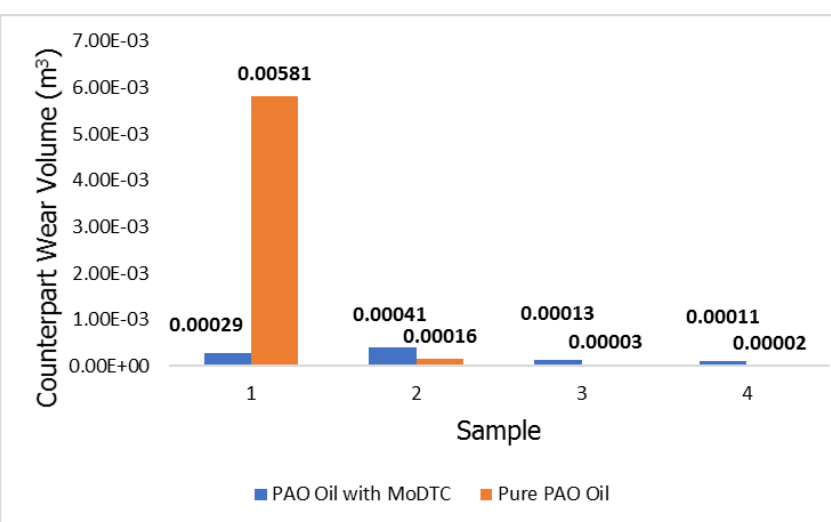

Fig. 7 To compare with wear volume of Counter materials $\left(\mathrm{Si}_{3} \mathrm{~N}_{4}\right.$ ball) under the PAO-4 base Oil and PAO-4 with MoDTC

However, in the case of $\mathrm{Cu}$ doped ta- $\mathrm{C}$ coatings, when the $\mathrm{Cu}$ sputtering power was $50 \mathrm{~W}$, the disk wear decreased slightly, but at $100 \mathrm{~W}$ and $200 \mathrm{~W}$, the wear of the counterpart material decreased sharply. This phenomenon shows that the coating with higher sputter power has better wear resistance when lubricated with PAO-4 base oil.

More interestingly, in the PAO-4 oil with MoDTC lubrication, low wear amount was observed in $\mathrm{Cu}$ doped ta-C coatings including ta-C coatings and counterpart materials. In addition, as the $\mathrm{Cu}$ sputtering power increases, the amount of disk wear increases, and the lowest disk wear behavior is seen in the general ta-C coating. This might be due to tribo-chemical reaction in Si3N4 that resulted in low wear under the boundary lubrication [10].
In this research, the addition of MoDTC in PAO-4 base oil showed positive effects on the wear performance. The wear rate in both ta-C/Si3N4 and $\mathrm{Cu} / \mathrm{ta}-\mathrm{C} / \mathrm{Si} 3 \mathrm{~N} 4$ contacts displayed similarly same behaviour as it resulted low wear under PAO oil with MoDTC lubrication. The wear rate for all types of DLCs in PAO oil with MoDTC are low suggesting that even with the presence of MoDTC, the wear rate is rather controlled by the nature of the DLC itself than the MoDTC [8].

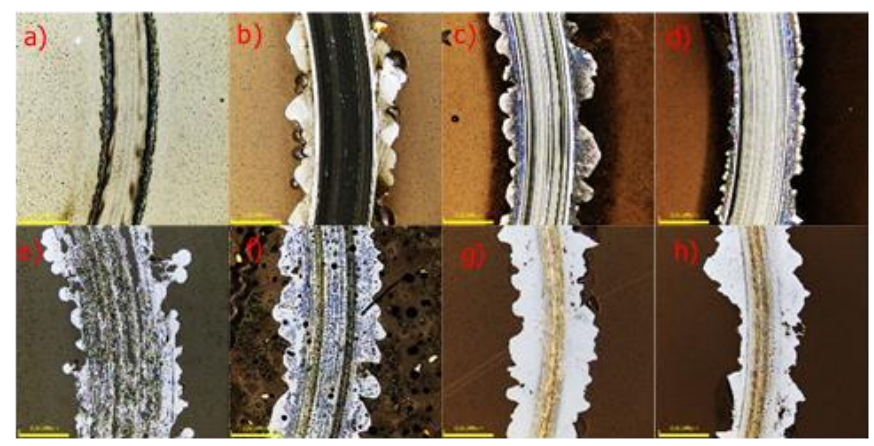

Fig. 8 Wear track of coatings for a) ta-C b) Cu-doped ta-C $(50 \mathrm{~W}) \mathrm{c}) \mathrm{Cu}$-doped ta-C (150W) and d) Cu-doped ta-C (200W) under PAO-4 Oil with MoDTC and e) ta-C f) $\mathrm{Cu}$-doped ta-C (50W) g) Cu-doped ta-C (150W) and h) Cu-doped ta-C (200W) under PAO-4 Base Oil

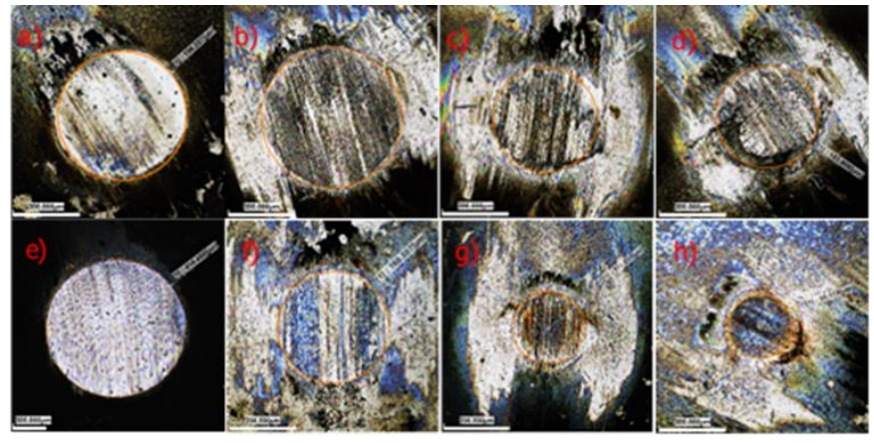

Fig. 9 Wear scar of the counterpart material for a) ta-C b) $\mathrm{Cu}-$ doped ta-C (50W) c) Cu-doped ta-C (150W) and d) Cu-doped ta-C (200W) under PAO-4 Oil with MoDTC and e) ta-C f) $\mathrm{Cu}-$ doped ta-C (50W) g) Cu-doped ta-C (150W) and h) $\mathrm{Cu}$-doped ta-C (200W) under PAO-4 Base Oil

\subsection{Surface Analysis}

The wear track image (Fig. 8) on the coating surface and the wear scar of the counterpart material (Fig. 9) were observed by using the laser scanning confocal microscope. It can be observed that the wear tracks on the coating surface of all samples are severely damage when lubricated with pure PAO oil. The wear width of Sample 1 (ta-C coating) in pure PAO oil is significant due to abrasive wear. This evidence of abrasive wear can be evidence in the friction and wear phenomena of the ta-C coating as mentioned above.

Meanwhile, the wear track on the coating surface of Sample 1 (ta-C coating) is the smoothest when lubricated under PAO oil with MoDTC. This might be due to less formation of wear debris in ta-C coating than $\mathrm{Cu} /$ ta-C coating. In Fig. 10 and Fig. 11, the size of wear scar of the counterpart material reduced as the sputter power increased in $\mathrm{Cu} / \mathrm{ta}-\mathrm{C} / \mathrm{Si} 3 \mathrm{~N} 4$ contacts for both types of lubrications. It can be observed that the size of wear scars of counterpart material for Samples $2(\mathrm{Cu} / \mathrm{ta}-\mathrm{C}, 50 \mathrm{~W}), 3(\mathrm{Cu} / \mathrm{ta}-\mathrm{C}$, $100 \mathrm{~W})$ and $4(\mathrm{Cu} / \mathrm{ta}-\mathrm{C}, 150 \mathrm{~W})$ are smaller under pure PAO oil lubrication compared to PAO oil with MoDTC. This might be 
due to the formation of tribo-film on the counterpart ball. However, it is the opposite in ta-C/Si3N4 contacts whereas size of wear scar of counterpart material is smaller under PAO oil with MoDTC lubrication compared to pure PAO oil.

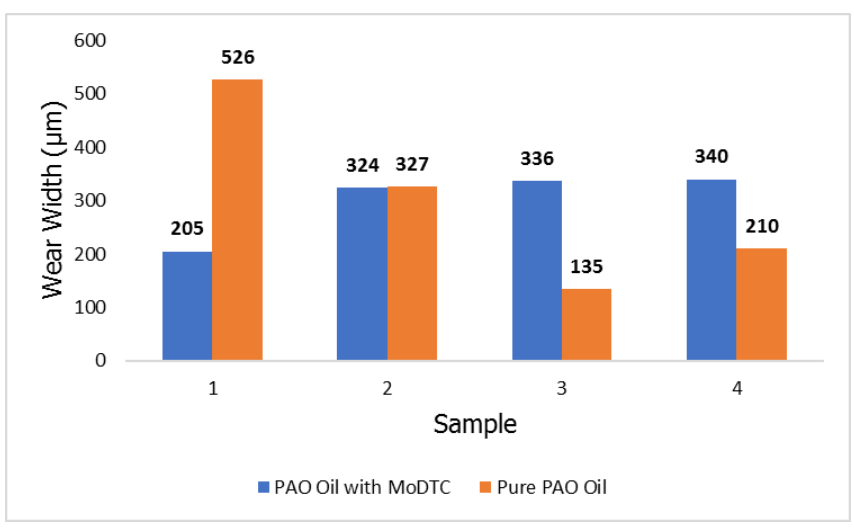

Fig. 10 Width of wear track of the coatings

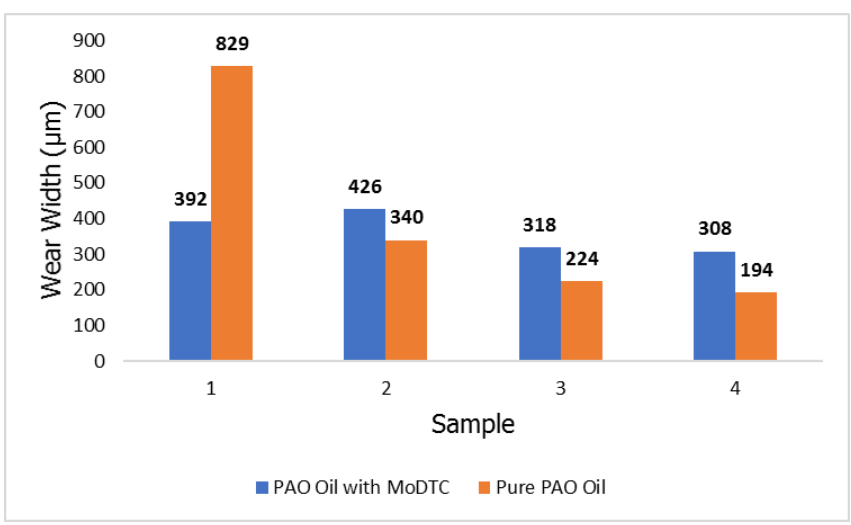

Fig. 11 Width of wear scar of the $\mathrm{Si}_{3} \mathrm{~N}_{4}$ balls

\section{Conclusion}

In this research, silicon wafers were coated with ta-C and $\mathrm{Cu} /$ ta- $\mathrm{C}$ coating with different sputter power. The sputter powers of $50 \mathrm{~W}, 150 \mathrm{~W}$ and $200 \mathrm{~W}$ were used for the $\mathrm{Cu} / \mathrm{ta}-\mathrm{C}$ coatings. All samples were examined for its frictional and wear behaviour when lubricated with two types of lubrications which are PAO-4 base oil and PAO-4 oil with MoDTC.

The results show that:

i. $\mathrm{Cu} / \mathrm{ta}-\mathrm{C}$ coating with lower sputter power has smaller friction coefficient but ta-C coating has the smallest when lubricated under PAO-4 oil with MoDTC.

ii. $\mathrm{Cu} / \mathrm{ta}-\mathrm{C}$ coating with higher sputter power has better wear resistance under PAO-4 base oil lubrication.

iii. The size of wear scar of the counterpart material reduced as the sputter power increase in $\mathrm{Cu} / \mathrm{ta}-\mathrm{C} / \mathrm{Si} 3 \mathrm{~N} 4$ contacts for both types of lubrications.

\section{References}

[1] Ren, Z., Qin, H., Dong, Y., Doll, G.L. and Ye, C., 2019. A boron-doped diamond like carbon coating with high hardness and low friction coefficient. Wear, 436, p.203031.

[2] Wang, X., Sui, X., Zhang, S., Yan, M., Yang, J., Hao, J. and Liu, W., 2019. Effect of deposition pressures on uniformity, mechanical and tribological properties of thick DLC coatings inside of a long pipe prepared by PECVD method. Surface and Coatings Technology, 375, pp.150-157.

[3] Ean, Y.C., Jang, Y.J., Kim, J.K., Hsien, W.L.Y., Siambun, N.J. and Kim, S.S., 2017. Effect of substrate bias on the tribological behavior of ta-C coating prepared by filtered cathodic vacuum arc. International Journal of Precision Engineering and Manufacturing, 18(5), pp.779-784..

[4] Dai, W., Wang, A. and Wang, Q., 2015. Microstructure and mechanical property of diamond-like carbon films with ductile copper incorporation. Surface and Coatings Technology, 272, pp.33-38.

[5] Jang, Y.J., Kang, Y.J., Kitazume, K., Umehara, N. and Kim, J., 2016. Mechanical and electrical properties of micron-thick nitrogen-doped tetrahedral amorphous carbon coatings. Diamond and Related Materials, 69, pp.121-126.

[6] Yazawa, S., Minami, I. and Prakash, B., 2014. Reducing friction and wear of tribological systems through hybrid tribofilm consisting of coating and lubricants. Lubricants, 2(2), pp.90-112.

[7] Vengudusamy, B., Green, J.H., Lamb, G.D. and Spikes, H.A., 2012. Behaviour of MoDTC in DLC/DLC and DLC/steel contacts. Tribology International, 54, pp.6876.

[8] Zahid, R., Masjuki, H.H., Varman, M., Mufti, R.A., Kalam, M.A. and Gulzar, M., 2015. Effect of lubricant formulations on the tribological performance of selfmated doped DLC contacts: a review. Tribology Letters, 58(2), p.32.

[9] Lee, W.Y., Tokoroyama, T., Jang, Y.J. and Umehara, N., 2018. Effect of Substrate Bias and Temperature on Friction and Wear Properties for ta-C Coating Prepared under Different Substrate Bias Voltages with Filtered Cathodic Vacuum Arc Deposition. Tribology Online, 13(5), pp.241-247.

[10] Zeng, Q., Yu, F. and Dong, G., 2013. Superlubricity behaviors of Si3N4/DLC Films under PAO oil with nano boron nitride additive lubrication. Surface and interface analysis, 45(8), pp.1283-1290. 